\title{
Additive Manufacturing of AlSi10Mg Alloy Using Direct Energy Deposition: Microstructure and Hardness Characterization
}

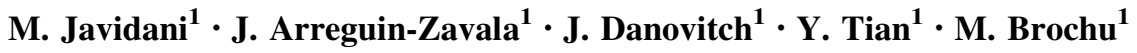

Submitted: 2 April 2016/in revised form: 9 September 2016/Published online: 8 December 2016

(C) ASM International 2016

\begin{abstract}
This paper aims to study the manufacturing of the AlSi10Mg alloy with direct energy deposition (DED) process. Following fabrication, the macro- and microstructural evolution of the as-processed specimens was initially investigated using optical microscopy and scanning electron microscopy. Columnar dendritic structure was the dominant solidification feature of the deposit; nevertheless, detailed microstructural analysis revealed cellular morphology near the substrate and equiaxed dendrites at the top end of the deposit. Moreover, the microstructural morphology in the melt pool boundary of the deposit differed from the one in the core of the layers. The remaining porosity of the deposit was evaluated by Archimedes' principle and by image analysis of the polished surface. Crystallographic texture in the deposit was also assessed using electron backscatter diffraction and $\mathrm{x}$-ray diffraction analysis. The dendrites were unidirectionally oriented at an angle of $\sim 80^{\circ}$ to the substrate. EPMA line scans were performed to evaluate the compositional variation and elemental segregation in different locations. Eventually, microhardness (HV) tests were conducted in order to study the hardness gradient in the asDED-processed specimen along the deposition direction. The presented results, which exhibited a deposit with an almost defect free structure, indicate that the DED process can suitable for the deposition of Al-Si-based alloys with a highly consolidated structure.
\end{abstract}

M. Brochu

mathieu.brochu@mcgill.ca

1 Mining and Materials Engineering, McGill University, 3610 University Street, Montreal, QC H3A 0C5, Canada
Keywords additive manufacturing $\cdot \mathrm{AlSi} 10 \mathrm{Mg}$. aluminum alloys $\cdot$ direct energy deposition

\section{Introduction}

Additive manufacturing (AM) is a process used to build three-dimensional objects by adding layers of material from a computer-aided design (CAD) model. AM techniques can build up parts into geometries that would otherwise be impossible to fabricate by conventional manufacturing methods. This technique differs from conventional manufacturing methods which are based on removing material from a solid block to create the final part (Ref 1).

AM is one of the most rapidly growing technologies of the last few years (Ref 2), with the aerospace and medical industries providing a strong impulse toward the research of metal AM processing. Due to their industrial interest, the metal AM research has mainly been focused on Ti6Al4V, stainless steel and Inconel as starting materials (Ref 3). On the other hand, due to their outstanding physical properties, such as low weight, excellent oxidation resistance, good mechanical properties, and high thermal and electrical conductivity, aluminum and its alloys are the second most used metals in the world (Ref 4).

The main focus of the reported AM studies is on Al-Si casting alloys, which are suitable for welding. Recently, significant efforts to develop and optimize their AM deposition process by using powder bed fusion laser (PBFL) and direct energy deposition (DED) technologies have been done (Ref 5,6). Direct energy deposition is a technology that produces fully dense, functional metal parts by depositing metal powders using a laser beam as energy source and a closed-loop control system to maintain 
dimensional accuracy and material integrity (Ref 6). This technology is used to repair, by rebuilding sections of worn or damaged components, and to manufacture new components. One of the major challenges of this material is its high reflectivity which causes a low rate of energy absorption promoting consolidation defects such as pores, cracks and unmelted areas (Ref 7).

The conventional cast Al-Si hypo-eutectic alloys are generally solidified with dendritic structure and composed of primary $\alpha$-Al plus Al-Si eutectic phase. However, the solidification features and the microstructural morphology are strongly influenced by process parameters, especially solidification rate (Ref 8,9). For instance, the solidification feature of the specimens processed with selective laser melting (SLM) is generally dominated with cellular morphology (Ref 5, 10). Dinda et al. (Ref 6), using AlSi11 alloy processed with direct melt deposition, obtained a banding microstructure in such a way that the layers of the deposit were initially solidified with cellular morphology, but further away from the substrate, in particular after the tenth layer, a dendritic morphology would dominate. The solidification feature and microstructural morphology of specimens must be taken into account, since they strongly affect the subsequent mechanical properties. For instance, the microhardness of AlSi10(Mg) alloys processed with SLM is about $130 \mathrm{HV}$ (Ref 11, 12), but for the conventional cast alloys, a HV of 55 has been reported (Ref 8). Dinda et al. (Ref 6), for the direct melt deposit specimen, obtained a HV of 107.

The aim of this study is to characterize AlSi10Mg alloy parts made using DED technology. During this work, the solidification features and morphology, microstructure development, area percentage of porosity, crystallographic texture orientation, elemental segregation and hardness of the AlSi10Mg alloy manufactured by DED process are elaborated upon.

\section{Experimental Methods}

The DED parts were printed using a laser fiber $(500 \mathrm{~W}$ IREPA, IPG Photonics Corporation, Ilkirch, France) coupled with a powder hopper (Medicoat powder feeder, Medicoat AG, Mägenwil, Switzerland). An angle of $10^{\circ}$ was maintained between the deposition nozzle and the build plane to avoid back reflection. A time delay was imposed between each pass to maintain similar solidification throughout the build and to avoid slumping of the deposit. Figure 1 schematically demonstrates the deposition pattern and the laser scan direction. The manufacturer used proprietary process parameters to produce the DED parts. A commercially available AlSi $10 \mathrm{Mg}$ powder, with the chemical composition presented in Table 1, was used in

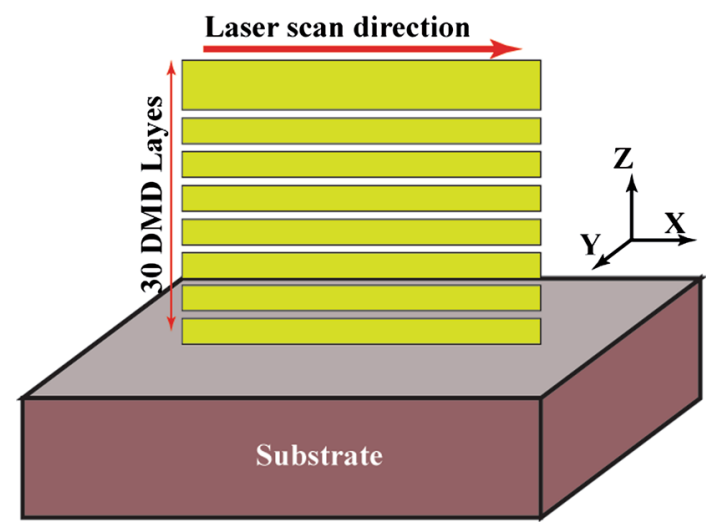

Fig. 1 Schematic illustration of deposition pattern and laser scan direction

Table 1 Chemical composition of the AlSi10Mg powder (wt.\%)

\begin{tabular}{ccccccccc}
\hline $\mathrm{Si}$ & $\mathrm{Fe}$ & $\mathrm{Cu}$ & $\mathrm{Mn}$ & $\mathrm{Mg}$ & $\mathrm{Zn}$ & $\mathrm{Ni}$ & $\mathrm{Ti}$ & $\mathrm{Al}$ \\
\hline 9.6 & 0.16 & 0.003 & $<0.001$ & 0.4 & $<0.002$ & $<0.005$ & 0.004 & $\mathrm{Bal}$. \\
\hline
\end{tabular}

this study. A particle size distribution ranging between 45 and 90 microns was selected for its compatibility with the deposition unit.

Standard metallographic procedures were used for macro- and microstructural characterization in the longitudinal sectioned (X-Z) specimens. A light optical microscope (OM) (EPIPHOT 200, Nikon, Tokyo, Japan) with Clemex Vision System and a SEM (SU3500, Hitachi, Tokyo, Japan) equipped with electron backscatter diffraction (EBSD) system (Oxford Instruments Nordlys Nano EBSD detector using AztecHKL software) were used to evaluate the microstructures. Acquisition of EBSD data was done at an accelerating voltage of $20 \mathrm{kV}$ and a step size of $5 \mu \mathrm{m}$. The EBSD data were analyzed using HKL Channel5 software. Keller's reagent was used for etching the metallographic samples.

X-ray diffraction (XRD) (D8 Discover, Bruker, Billerica, Massachusetts, USA) was performed using $\mathrm{Cu}$-source system at $40 \mathrm{kV}$ and $40 \mathrm{~mA}$. All samples were scanned from a $2 \theta$ of $10^{\circ}$ to $102^{\circ}$ ( 4 frames with frame width of $23^{\circ}$ at $300 \mathrm{~s} /$ frame). Measurements were taken at three points (bottom, middle and top) on a polished longitudinal (X-Z) sectioned specimen.

The bulk density of as-DED-processed specimens was measured using Archimedes' principle, to quantify the volume fraction of porosity (Ref 13,14$)$. The density measurements involved weighing polished specimens in air and then immersing them in distilled water. The densities of the specimens were derived from their mass and volume; the driven density was then compared to the theoretical density $\left(\sim 2.68 \mathrm{~g} / \mathrm{cm}^{3}\right)$ (Ref $\left.11,12,15\right)$ from which the volume fractions of porosity were calculated. The samples 
were weighed using an electronic balance (AT200, Mettler Toledo, Markham, Ontario, Canada) with an accuracy of $\pm 0.1 \mathrm{mg}$. Furthermore, the area percentage of porosity was measured by image analysis of optical micrographs taken from the longitudinal (X-Z) section. ImageJ software was utilized for image analysis of the deposited sample to measure secondary dendrite arm spacing (SDAS); this analysis was carried out systematically in the dendritic region from the lower to upper section of the sample. Measurements on at least four dendrites having minimum five SDAS were taken for each micrograph, and the averages with error bars are presented.

It has been reported that dendrite arm spacing in a given alloy depends significantly, and almost exclusively, on cooling rate and varies little from alloy to alloy (Ref 9). Therefore, the relationship of $\lambda=a \cdot R^{-n}$ was used to calculate the variation of the cooling rate versus distance from the substrate, where $\lambda$ is the SDAS $(\mu \mathrm{m}), R$ is the average cooling rate (K/s), and $a$ and $n$ are the material-dependent constants ( $a=47, n=0.33$ for eutectic Al-Si alloys) (Ref $6,16,17)$.

The quantitative analysis of the compositions of both the inter-dendritic regions and the dendritic cores was conducted on the line-scan measurements by means of an electron probe microanalysis (EPMA) (8900, JEOL, Tokyo, Japan). The standards of different elements were used for the EPMA measurement. The interaction volume for EPMA is around $20 \mu \mathrm{m}^{3}$ according to Monte Carlo simulations from Casino software (Ref 18).
The hardness of the deposit was measured by means of a microhardness indenter (Clark CM-100AT, SUN-TEC, Novi, Michigan, USA) using $10 \mathrm{~g}$ force and a $10 \mathrm{~s}$ indentation dwell time. Indentations were conducted in a longitudinal section of the specimen, systematically from bottom (near the substrate) to top end of the specimen.

\section{Results and Discussion}

\section{Solidification Structure and Microconstituents of the Specimen}

Figure 2 shows typical OM micrographs in the longitudinal section (X-Z) of the as-processed specimen in three different locations of the deposit: (a) bottom of the specimen (close to the substrate), (b) middle of the specimen and (c) top end. Although the dominant solidification structure was columnar dendrites, depending on the distance of the deposited layers from the substrate, three different solidification features were established across the specimen. The principal solidification feature near the substrate was cellular, which was progressively substituted by a columnar dendritic structure when further away from the substrate. Subsequently, in a narrow zone at topmost layer of the deposit, an equiaxed dendritic structure prevailed.

In order to gain better insight into the morphology, size and distribution of the microconstituents (most notably $\mathrm{Si}$ particles) in the deposit, SEM characterizations were

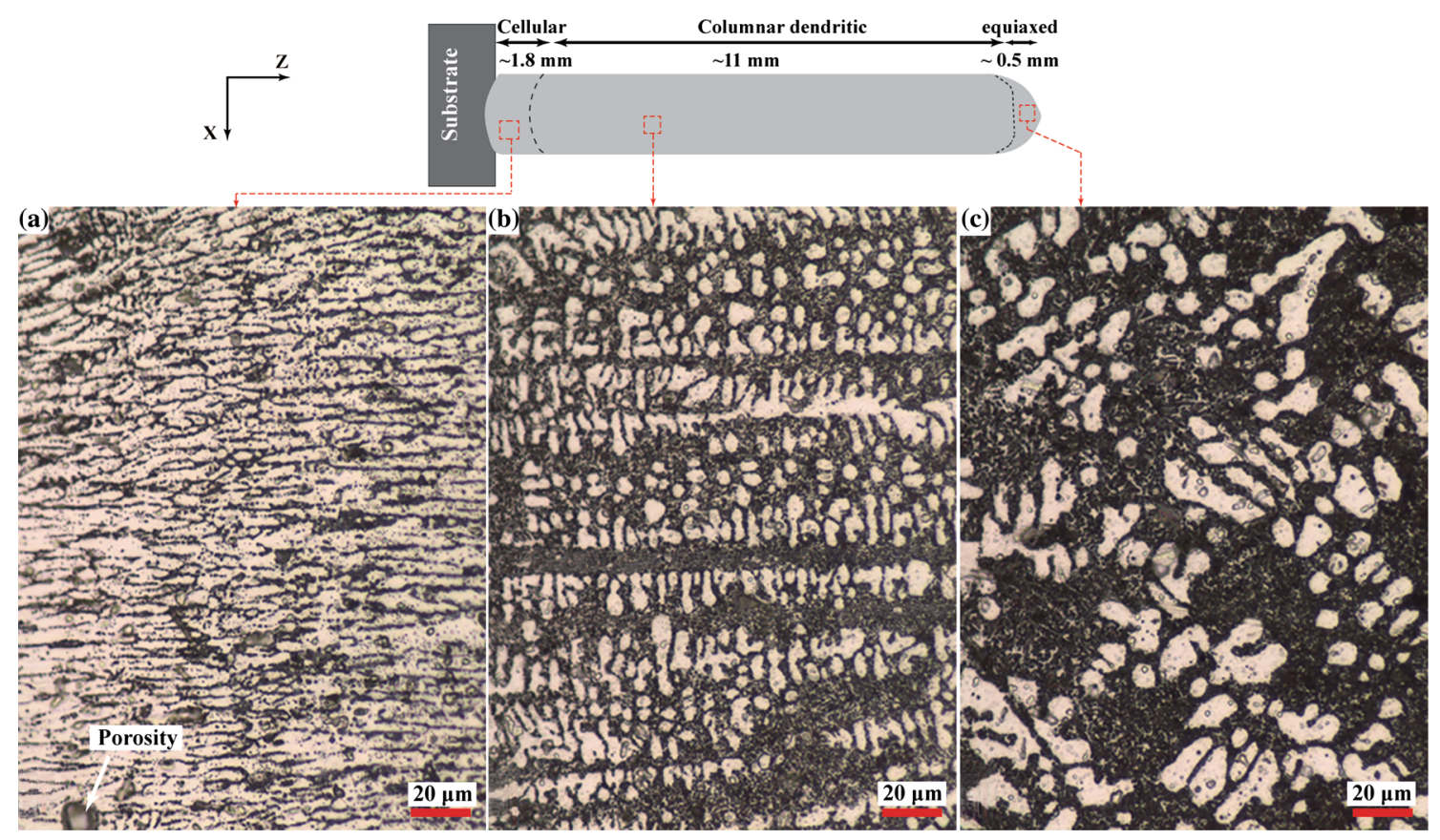

Fig. 2 Representative OM micrographs of the as-DED-processed specimen in longitudinal section (X-Z) 
carried out. Figure 3(a), (d), and (g) illustrates, respectively, the micrographs in cellular, columnar dendritic and equiaxed zones. In order to clearly elucidate the size and morphology of the microconstituents, Fig. 3(b-i) magnifies the dotted area in Fig. 3(a), (d), and (g). The cellular region (Fig. 3a-c) was composed of $\alpha$-Al cells surrounded by very fine inter-cellular Si particles and small micropores.

At the start of the deposition process, the base plate acts as a heat extractor for the first layers. As the number of deposited layers increases, the influence from base plate becomes negligible. This phenomenon is discussed in detail at the following section. Due to the progressive decrease in the cooling rate from the bottom part to the top part of the deposit, a smooth and gradual transition of solidification features from cellular to columnar dendritic was established roughly after the first 5 deposited layers. As shown in Fig. 3(d-f), the columnar dendritic structure consisted of an $\alpha$-Al matrix, eutectic Si particles with fibrous morphology, and randomly distributed spherical porosity with diameters of $\sim 7$ to $\sim 25 \mu \mathrm{m}$. Eventually, in the equiaxed dendritic region, alike the columnar dendritic region, the microconstituents were encompassed of an $\alpha$ - $\mathrm{Al}$ matrix and eutectic Si particles. However, the dendrites, instead of growing in a unified solidification direction, were observed to grow in multiple directions. Moreover, the size of the Si eutectic particles in the equiaxed region was much greater than those in the dendritic region. As clearly shown in Fig. 2 and 3, the overall microstructure was more refined at the bottom (compared to the top) of the deposit.

\section{Effect of Heat Release on Solidification Structure}

As described above, the microstructure of the transverse section varies at different locations of the deposit depending upon the local cooling rate and the heat flow direction. The growth of the dendrites is opposite to the resultant heat flow direction (Ref 3, 19, 20). In the DED process, heat of the melt flows mostly through the substrate or previously deposited layers; however, it can also be partially extracted through the adjacent solidified deposit layer which grew from the trailing end of the melt pool, and/or via the surrounding atmosphere. The heat extraction through the substrate (or through the already deposited layers) imposes heat release by conduction, whereas the heat extraction through the adjacent deposited layer imposes heat release by convection. Depending on the location of melt in DED process, one or a combination of the aforementioned heat release processes dominates (Ref 19-21).

In the bottom section of the deposit, the heat of the melt is fully extracted via the substrate; therefore, the aligned dendritic/cellular feature is grown opposite to the heat flow direction and perpendicular to the solid/liquid interface (Ref 19, 21). By progressively getting further away from the substrate, the role of the adjacent solidified deposit layer (grown from the trailing end of the laser melt pool) in heat release will be gradually enhanced. Since the temperature of the deposit at the back of the melt pool is relatively high compared to the substrate, the resultant heat flow direction will be at an angle of more than $45^{\circ}$ and less than $90^{\circ}$ to the substrate (Ref 19, 21). As shown in Fig. 3, in the columnar dendritic region (at about half height of the deposit), the dendrites were unidirectionally oriented at an angle of $80 \pm 5^{\circ}$ to the substrate.

Eventually, at the upper part of the deposit (as shown in Fig. $3 \mathrm{~g}$ ), the columnar growth of the dendrites is suddenly substituted by equiaxed dendrites. As reported in the literature (Ref 19,21), the orientation transition is generally situated at the uppermost part of each layer, though, due to the partial remelting of the upper part of the previously deposited layer, this transition layer is eliminated from the microstructure of all the deposited layers except the last one. This orientation alteration is due to the effective role of the back of the melt pool and surrounding atmosphere (convection) in heat release at the top surface of the melt pool.

\section{Microstructure in the Boundaries of the Layer (Melt Pool)}

Detailed microstructure characterization, exemplified in Fig. 4(a-g), revealed that the size and morphology of the microconstituents ( $\alpha-\mathrm{Al}$ and Si eutectic) in the melt pool boundaries of DED layers differed from the ones in the core of the layers. The overall distinctive characteristic of the microconstituents in the boundaries were coarser microstructure (most notably $\mathrm{Si}$ particles) compared to ones in the core of the layers. Figure 4(a) and (d) illustrates the microstructural features in the boundaries in the bottom and middle sections of the columnar dendritic regions, respectively. Figure 4(b), (c), and (e-g) represent the magnified micrographs of the dashed/dotted rectangular regions in Fig. 4(a) and (d), respectively.

As shown in these micrographs, the solidification features were altered from the lower part (Fig. 4a) toward the upper part (Fig. 4d) of the deposit in the columnar dendritic region. At the lower part, as illustrated in Fig. 4(a-c), cellular morphology was dominated in the boundaries, unlike the core of the layers which were prevailed by dendritic structure. After the first 4 layers in the columnar dendritic region, the cellular feature in the boundaries was progressively substituted by dendritic morphology. An almost similar transition of morphology (banded structure) has been reported in the literature (Ref 6, 19); Dinda et al. (Ref 6) also stated that the cellular morphology was restricted to 

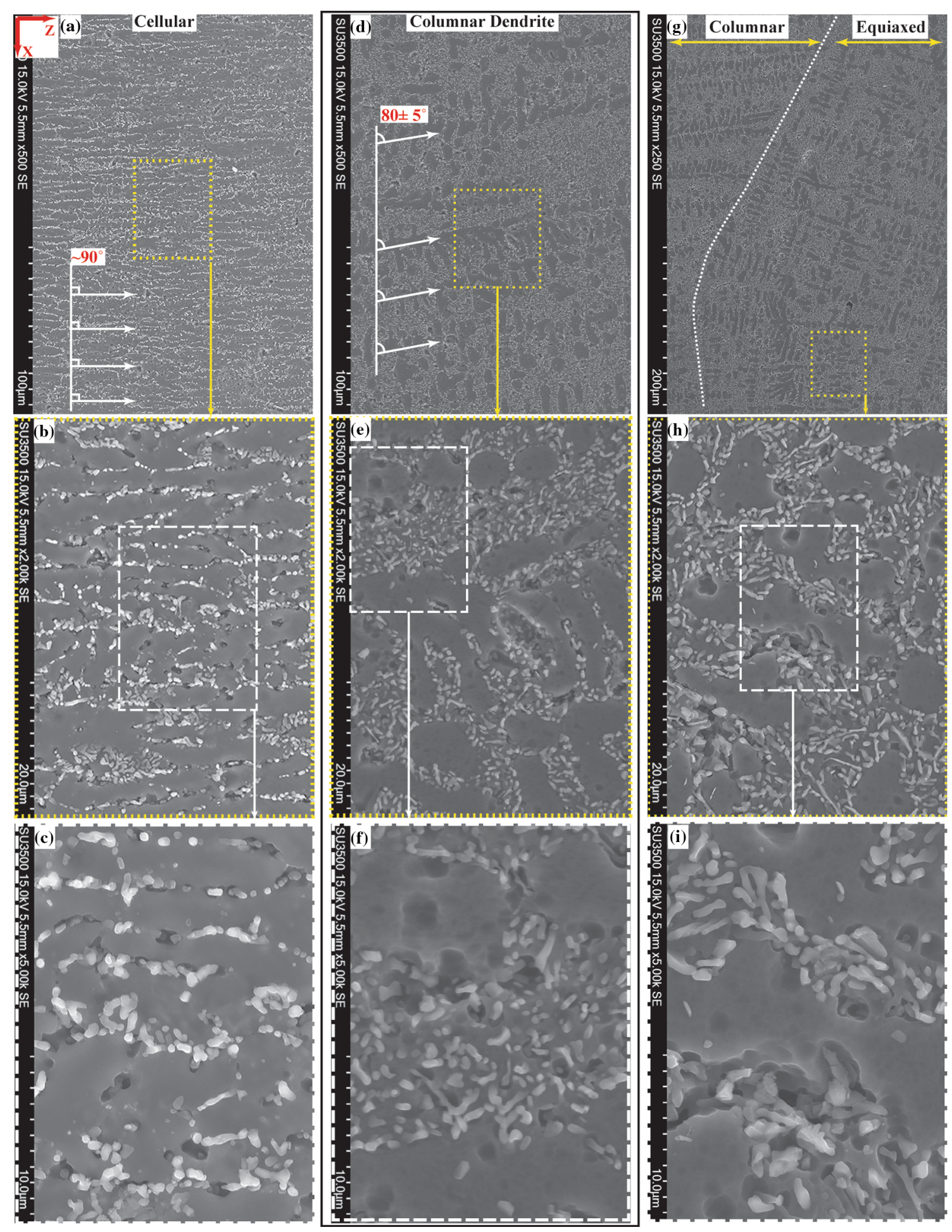

Fig. 3 Representative SEM micrographs of as-DED-processed specimen

the first ten layers of deposition. As shown in Fig. 4(d-g), a dendritic morphology with coarsened SDAS was dominant in the boundaries. In addition, as compared in these micrographs, the morphology and size of the Si eutectic particles were altered. The Si particles were much coarser in the boundaries and appeared with cubic-like morphology; however, in the core of the layers, they were finer and appeared with fibrous morphology. Changing the cooling 


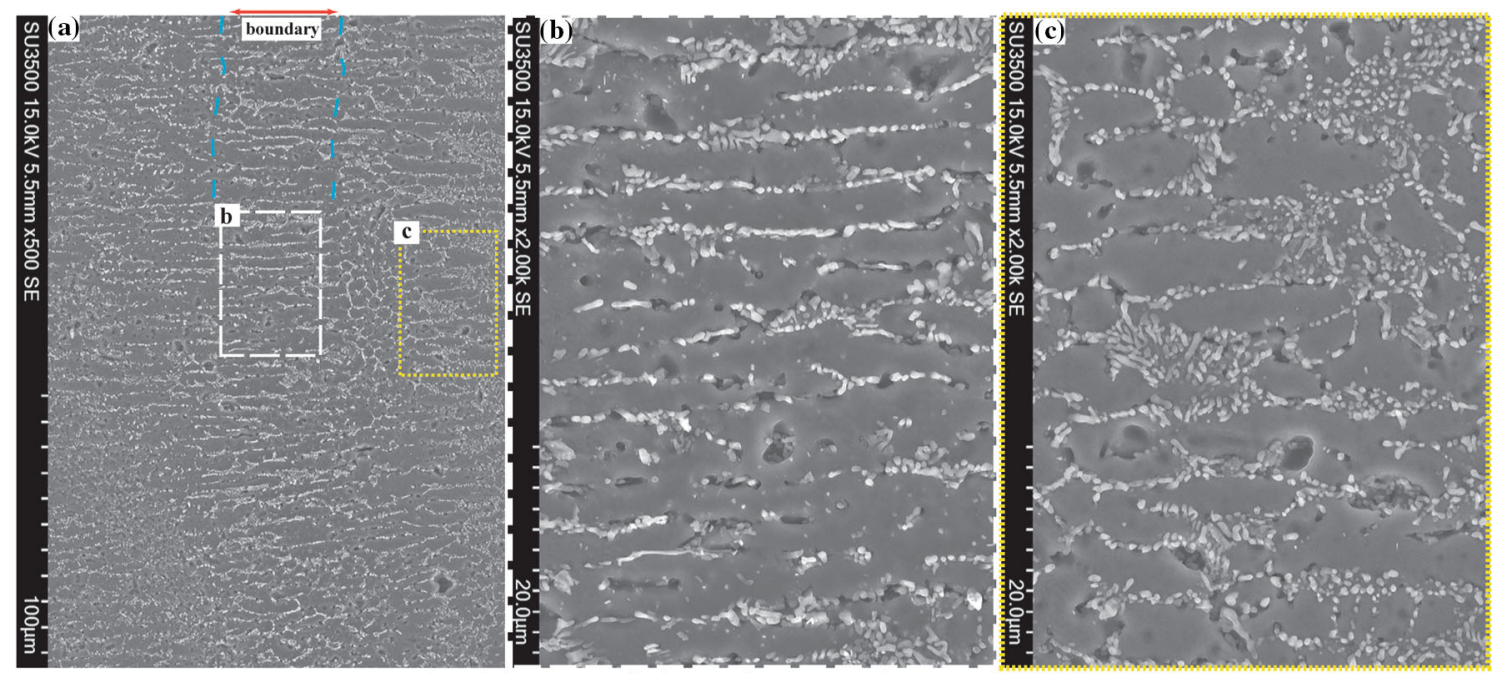

Lower section (close to cellular region ) of columnar dendritic region.
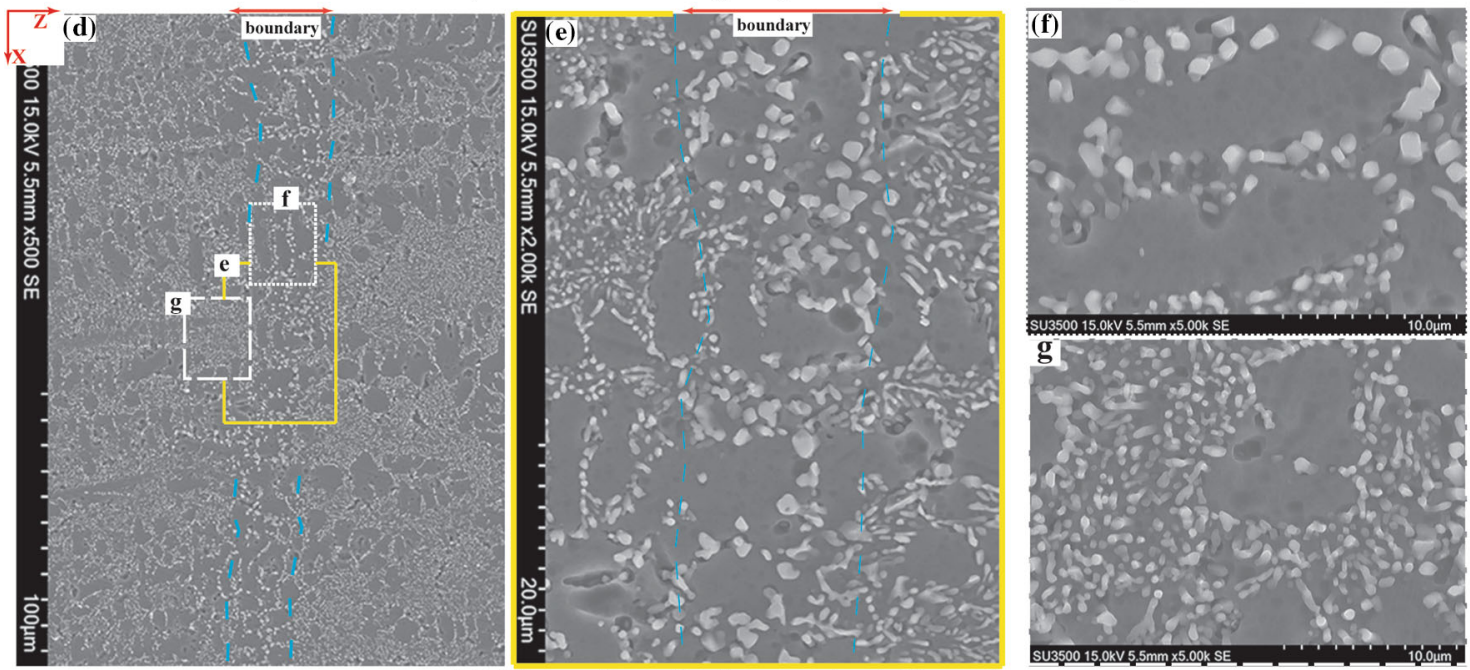

Middle section of columnar dendritic region.

Fig. 4 Boundaries of layers in dendritic region

rate from bottom layers of the deposit toward the top, which is detailed in the following section, implies alteration of the solidification feature.

The solidification in the new deposited layer always proceeded epitaxially, which is due to the predominant nucleation direction of the previously deposited layer. Due to the heat flow direction and the previous layer acting as a crystallographic seed, solidification always progressed with the same manner and the same dendritic growth direction as the previous layer.

\section{Porosity and Density of the Deposit}

The measured density value of the as-DED-processed specimen, by Archimedes' principle, was $\sim 2.67 \mathrm{~g} / \mathrm{cm}^{3}$. This value is $\sim 99.6 \%$ of the theoretical bulk density of the alloy $\left(\sim 2.68 \mathrm{~g} / \mathrm{cm}^{3}\right)$ (Ref 11, 12). Furthermore, the area percentage of porosity was also verified by image analysis in the polished longitudinal sectioned specimen; it was found that the pore area was $0.36 \pm 0.03 \%$ (i.e., $99.64 \pm 0.03 \%$ of the theoretical density). The measured density values, from both Archimedes' principle and image analysis, are evidence of a highly consolidated specimen. Moreover, macrostructural examination conducted on the polished specimens revealed neither the presence of macropores nor macrosegregation across the longitudinal section.

\section{SDAS and Cooling Rate}

A careful examination of the microstructure in the dendritic region of the specimen exhibited variations on SDAS. The measured SDAS values versus distance of the dendrites from the substrate are presented in Fig. 5. As shown, the SDAS values varied between $\sim 3.1$ and $\sim 5 \mu \mathrm{m}$ from bottom to top of the deposit (in the columnar dendritic region). 


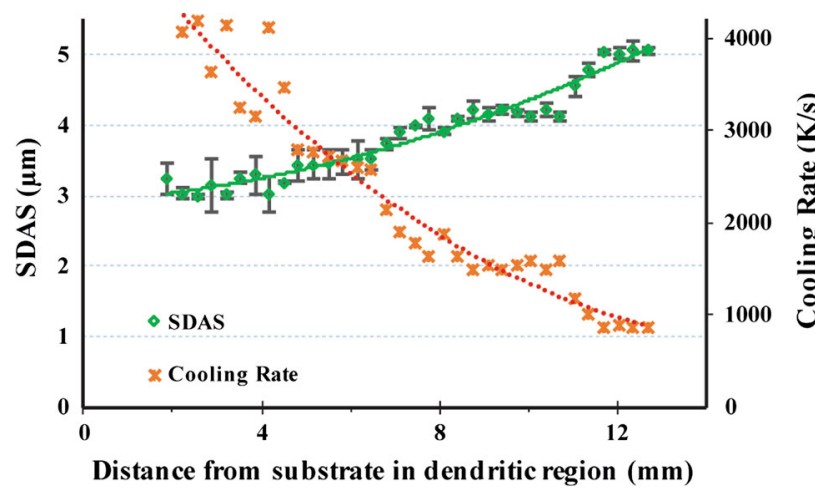

Fig. 5 SDAS and cooling rate vs distance from substrate
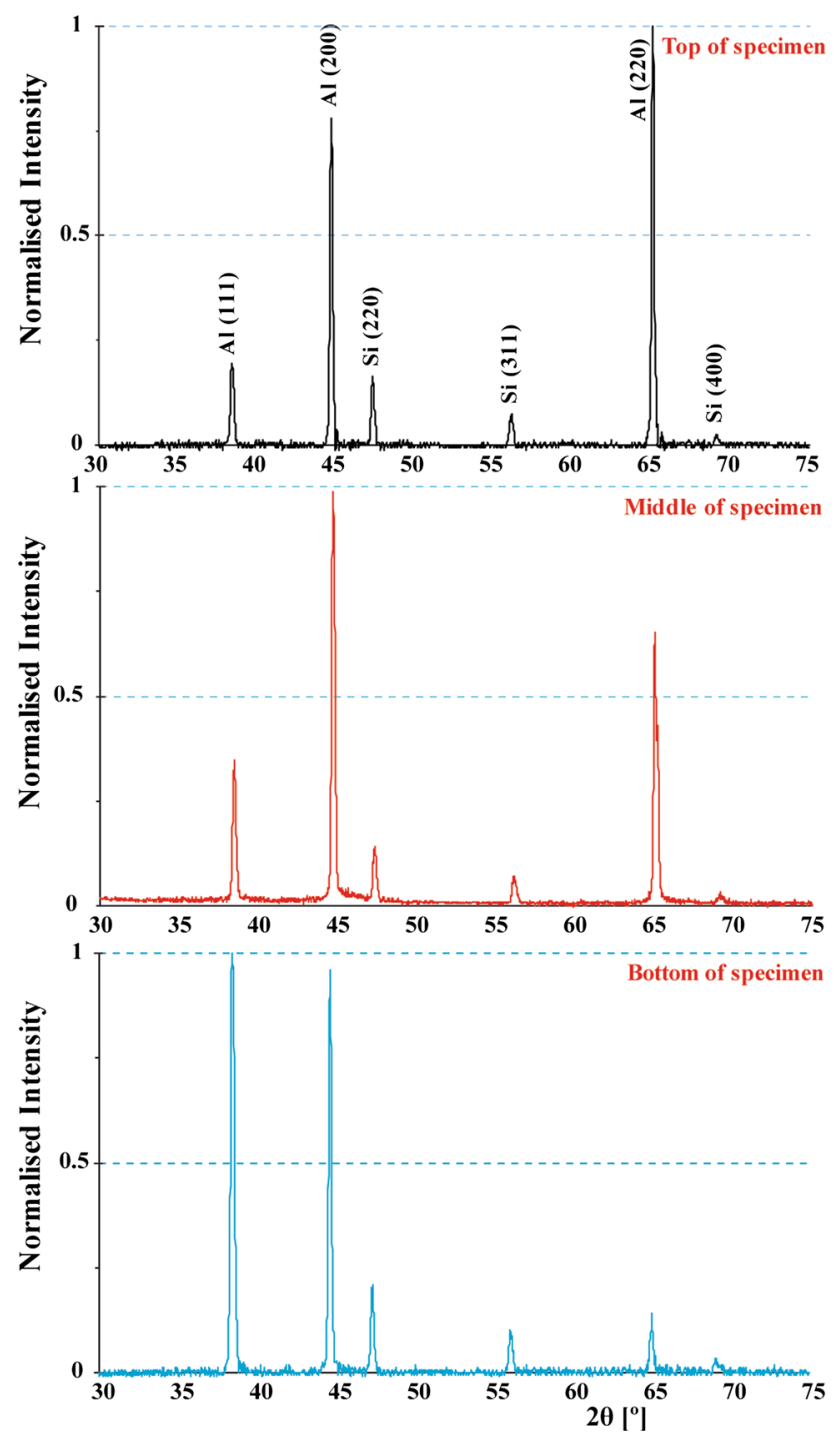

Fig. 6 XRD pattern comparison at top, middle (half height) and bottom sections of as-DED-processed specimen

The relationship of $\lambda=a \cdot R^{-n}$, detailed in "Experimental Methods" section, was used to calculate the variation of the cooling rate versus distance from the substrate.

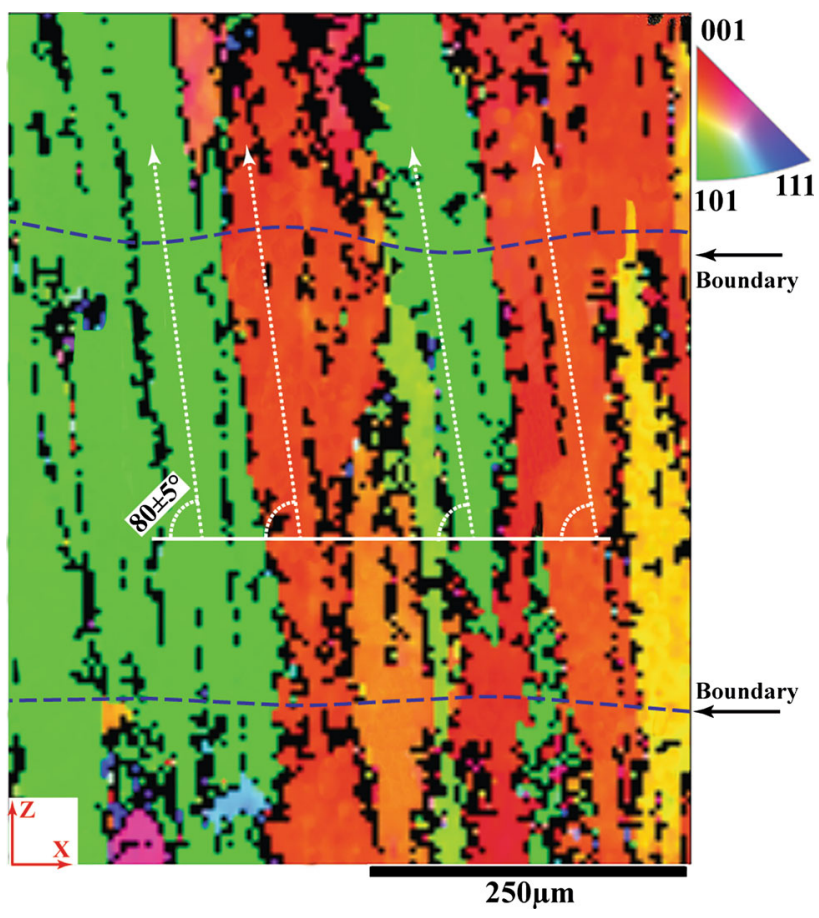

Fig. 7 EBSD mapping at middle (half of the height) of the specimen; inverse pole figure (IPF) colored orientation image map, with IPF

As presented in Fig. 5, the cooling rate during the DED process varied from $\sim 4000 \mathrm{~K} / \mathrm{s}$ in the bottom to $\sim 855 \mathrm{~K} /$ $\mathrm{s}$ in the top of the deposit. These values strongly differ with the ones in the conventional casting process which normally are in the range below $0.833 \mathrm{~K} / \mathrm{s}$.

\section{Crystallographic Texture Orientation (XRD and EBSD Results)}

As previously mentioned, the equilibrium microstructure of the specimen is a mixture of $\alpha$ - $\mathrm{Al}$ dendrites and eutectic Al-Si phase. XRD patterns were separately collected from the top, middle (half height) and bottom of the longitudinal section (X-Z) of the as-DED-processed specimen. The peak intensities between the top, middle and bottom locations are compared in Fig. 6. As shown, the presence of silicon particles throughout the specimen is observed. The relative intensities of the observed silicon peaks do not differ significantly from the top to the bottom, indicating that there is no large-scale segregation of the silicon within the sample. Moreover, there is no visible shift in the overall crystallographic orientation of the silicon precipitates with sample height. However, the aluminum peak intensities vary greatly with sample height. As shown in Fig. 6, the bottom section of the specimen appears to favor the (111) and (200) crystallographic orientations with more or less equal intensities, while in the middle and the top sections, there is a noticeable drop in intensities of both the (111) 
direction, favoring instead the (220) direction, which was nearly absent in the bottom section, and the (200) direction. Figure 6 indicates the formation of a strong texture during laser deposition process.

Electron backscattered diffraction (EBSD) was used to evaluate the grain size and crystallographic orientation in the deposits. Figure 7 represents the orientation image map, using inverse pole figure coloring, of the middle section of the deposit. As shown in this figure, the long axis (growth direction) of the columnar Al grains is oriented at $<001>$ and $<101>$ directions with an angle of $\sim 80^{\circ}$ toward the substrate. Due to the epitaxial growth nature, the $\mathrm{Al}$ grains in the newly deposited layers, as shown in Fig. 7, grew with the same crystallographic orientation of the $\mathrm{Al}$ grains in the previous layers. The EBSD results are in good agreement with the XRD results presented in Fig. 6.

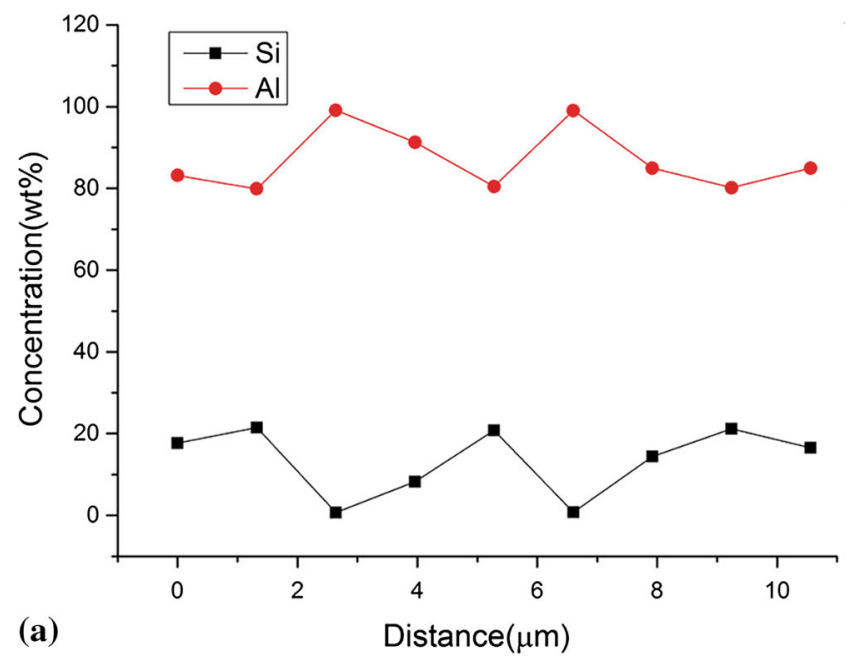

\section{Elemental Segregation}

EPMA line scans were performed to quantitatively obtain compositional variation and elemental segregation in different locations. Figure $8(\mathrm{a}-\mathrm{c})$ presents the EPMA linescan results in cellular, columnar dendrites and equiaxed dendrite regions, respectively. Comparable and good distribution of $\mathrm{Al}$ and $\mathrm{Si}$ across the longitudinal section (from bottom to top of the specimen) illustrates the presence of no macrosegregation all over the deposit, which is in agreement with the XRD results. Nevertheless, severe microsegregation of Si (up to $20 \mathrm{wt} . \%$ ) was detected where the corresponding Al distribution was varied from 99 to 80 wt. $\%$.

Based on the Scheil model (Ref 22) and Brody-Flemings model (Ref 23) with the back-diffusion theory, the ClyneKurz model (Ref 24) predicted the segregation pattern with

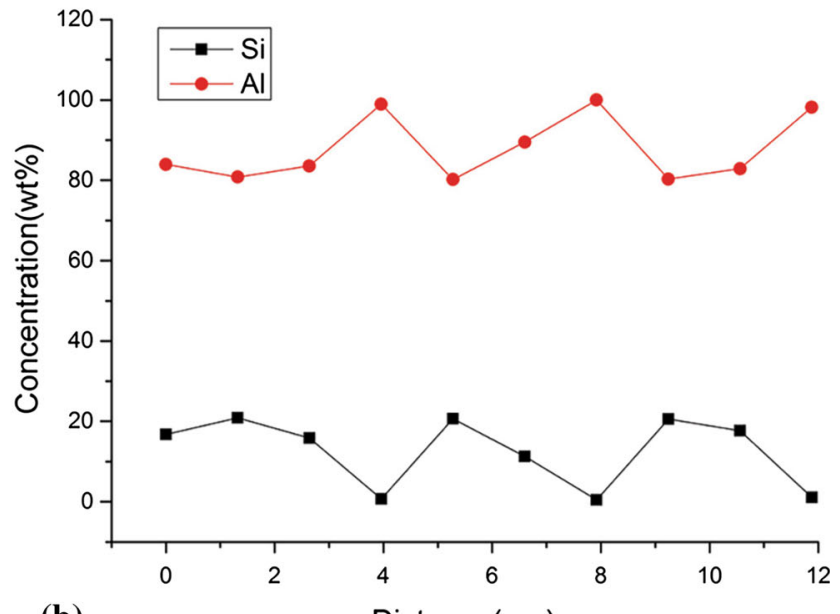

(b)

Distance $(\mu \mathrm{m})$

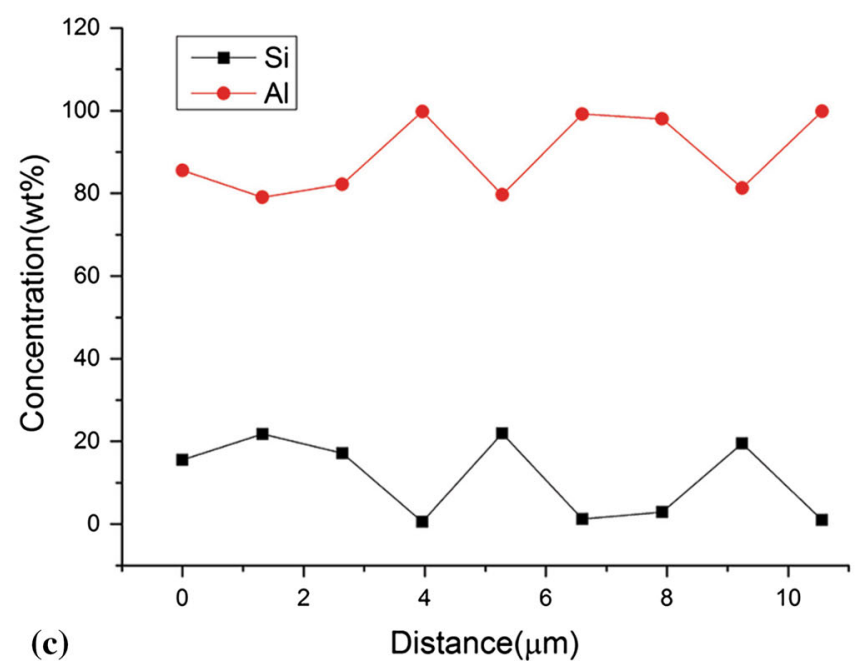

Fig. 8 EPMA line-scan results in (a) cellular microstructure in bottom; (b) dendritic microstructure in middle; and (c) equiaxed microstructure in top 


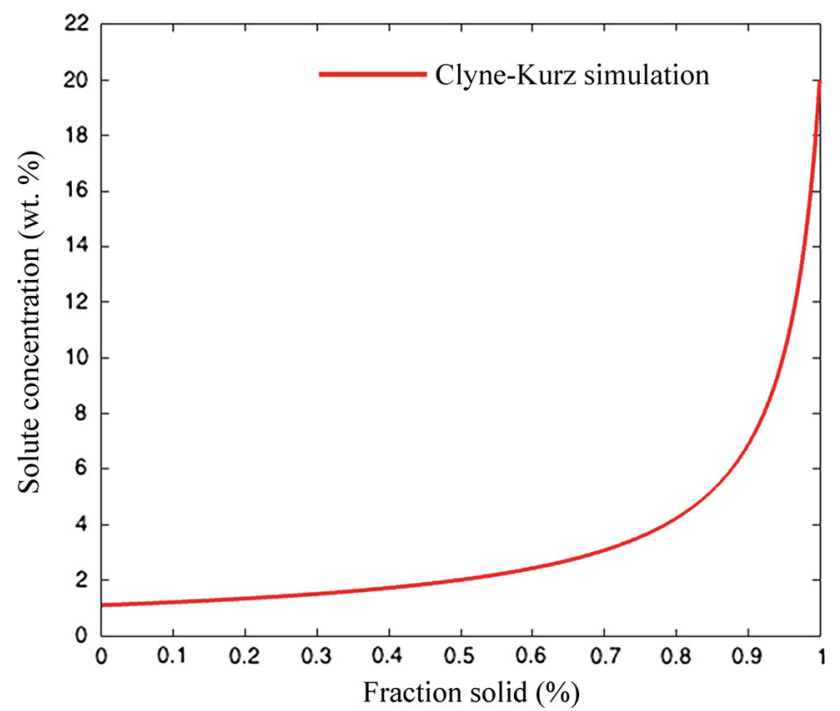

Fig. 9 Clyne-Kurz simulation of Si segregation

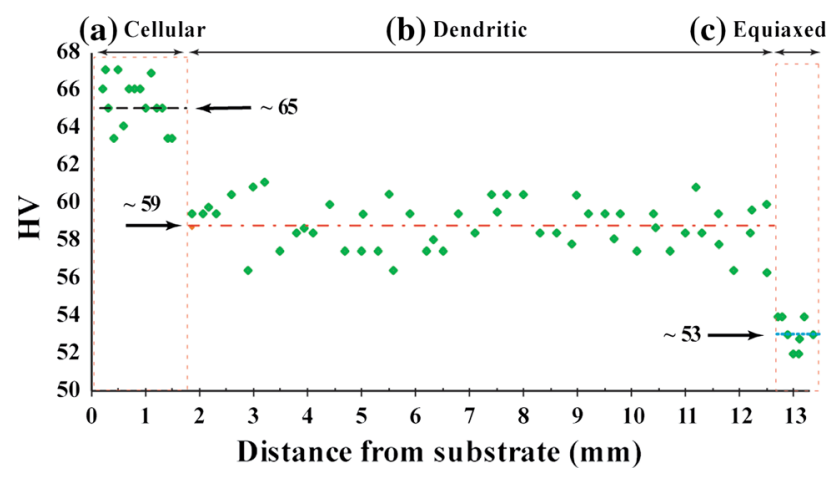

Fig. 10 Variation of microhardness all over of the specimen from bottom to top end

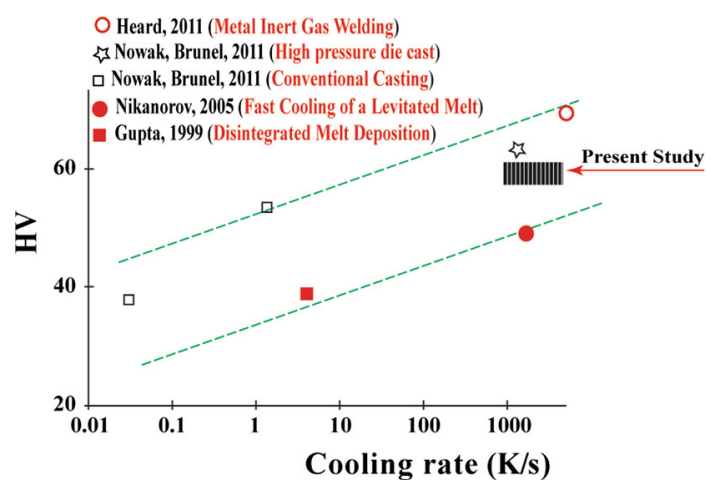

Fig. 11 Variation of Vickers hardness with cooling rate (Ref $8,13,17,26)$

corrected back-diffusion parameter for elemental segregation. The Clyne-Kurz model is described in Eq 1 through 3:

$C_{s}=k C_{0}\left[1-(1-2 \Omega k) f_{s}\right]^{(k-1) /(1-2 \Omega k)}$
$\Omega=\alpha[1-\exp (-1 / \alpha)]-1 / 2 \exp (-1 / 2 \alpha)$

$\alpha=\frac{D_{s} t_{f}}{L^{2}}=\frac{4 D_{s} t_{f}}{\lambda^{2}}$

where $C_{s}$ is the solute concentration in the solid at the solid-liquid interface, $k$ is the partition coefficient, $C_{0}$ is the nominal solute concentration, $f_{s}$ is the fraction of solid, $\alpha$ is a dimensionless back-diffusion parameter, $D_{s}$ is the diffusion coefficient of solute in the solid, $t_{f}$ is the local solidification time, $\lambda$ is dendrite arm spacing, while $L$ is half the dendrite arm spacing, and $\Omega$ is the correction function for $\alpha$. Values of $k=0.11$ and $\lambda=4 \mu \mathrm{m}(L=2 \mu \mathrm{m})$ were used (Ref 25), and $D_{s}=2.69 \times 10^{-11} \mathrm{~m}^{2} / \mathrm{s}$ that in solid phase was chosen (Ref 25). The cooling rate during solidification is required in order to measure $t_{f}$. Estimated by $G \times v$, the cooling rate during solidification is calculated to be in the order of $10^{3}{ }^{\circ} \mathrm{C} / \mathrm{s}$, and $t_{f}$ can be obtained accordingly.

The Clyne-Kurz result for $\mathrm{Si}$ segregation is shown in Fig. 9, where the predicted roughly $20 \mathrm{wt} . \%$ segregation of $\mathrm{Si}$ is in good agreement with the reported experimental observation. It also exhibits the feasibility of applying Clyne-Kurz simulation to calculate elemental segregation during the direct energy deposition process.

\section{Hardness}

Microhardness tests were conducted to determine the hardness gradient in the longitudinal section $(\mathrm{X}-\mathrm{Z})$ of the as-DED-processed specimen along the deposition direction. Figure 10 presents the variation of microhardness (HV) across the specimen from bottom to the top of the deposit. As shown in this figure, the deposit was classified into three major zones based on the presented hardness profile. The highest hardness value, with an average value of $\sim 65 \mathrm{HV}$, was obtained in the region near the substrate; as elaborated on earlier, this region was characterized by a fine cellular structure. By getting further away from the substrate and transitioning to the region with a dendritic structure, the microhardness was reduced to an average value of $\sim 59 \mathrm{HV}$. Despite the variation of SDAS in this region as presented in Fig. 5, the microhardness value remained more or less stable across the columnar dendritic region. By getting further away from the substrate and entering the region of equiaxed structure, the average microhardness value was reduced to $\sim 53 \mathrm{HV}$.

Figure 11 compares the average microhardness values for $\mathrm{AlSi}(\mathrm{Mg})$ alloys reported in the literature and obtained in the present study. Although the specimens in the selected references were processed with different conditions, they all solidified with a dendritic structure. As shown in this figure, the hardness of the $\mathrm{AlSi}(\mathrm{Mg})$ alloys 
present a wide range of variation, which strongly depend on the cooling rate of the melt. The hardness of the deposit is higher than the hardness of the conventional cast specimen and is close to the hardness value of the specimen produced with high-pressure die casting.

Nevertheless, the hardness of Al-Si alloys, beside the SDAS (cooling rate) of the specimen (Ref 26), strongly depends on microstructural morphology (size and distribution of microconstituents) as well (Ref 1). The refined scale of both the $\mathrm{Al}$ and Si phases results in higher hardness values. As mentioned earlier, the average microhardness values of the specimens processed with selective laser melting (SLM) are generally much higher than the ones obtained with the DED process. Rosenthal et al. (Ref 10) and Kempen et al. (Ref 11), respectively, reported $\sim 94$ and $\sim 127 \mathrm{HV}$ for AlSi10Mg specimens processed with SLM. Furthermore, Dinda et al. (Ref 6) obtained an average microhardness value of $\sim 107 \mathrm{HV}$ for laser-deposited Al-11\% Si specimen with cooling rate of $\sim 3.8 \times 10^{4} \mathrm{~K} / \mathrm{s}$ and average DAS of $1.4 \mu \mathrm{m}$. This increase in hardness is mainly lead by an Al cell refinement to $\sim 1 \mu \mathrm{m}$ (or less) throughout the printed sample.

\section{Conclusions}

- AlSi10Mg alloy was successfully printed with DED process. The printed deposits exhibited a highly consolidated and almost defect free structure.

- The dominant solidification structure of the deposit was columnar dendritic established parallel to the resultant heat flow direction, with $80 \pm 5^{\circ}$ to the substrate. Nevertheless, near the substrate cellular morphology, and at the top end of the deposits, equiaxed dendrites were prevalent.

- The section with cellular morphology displayed the highest hardness value ( $\sim 65 \mathrm{HV})$, while the uppermost section of the deposit with equiaxed dendritic feature showed the lowest hardness ( $\sim 53 \mathrm{HV})$. This weakness is attributed to the coarser microstructure compared to other counterpart.

- The size and morphology of the microconstituents in the melt pool boundaries of DED layers differed from the ones in the core of the layers. The overall distinctive characteristic of the microconstituents in the boundaries was a coarser microstructure compared to ones in the core of the layers.

- Line-scan analysis (with EPMA) did not show the presence of any macrosegregation across the longitudinal section, which was in agreement with the XRD results. Nevertheless, a severe microsegregation of $\mathrm{Si}$ element (up to $20 \mathrm{wt} . \%$ ) was detected. The experimental line-scan results were in good agreement with simulated results predicted by the Clyne-Kurz model.

Acknowledgments The authors would like to thank BeAM (France), for providing the samples.

\section{References}

1. R. Chou, J. Milligan, M. Paliwal, and M. Brochu, Additive Manufacturing of Al-12Si Alloy via Pulsed Selective Laser Melting, JOM, 2015, 67, p 590-596

2. W. Terry, Tracking Global Growth in Industrial-Scale Additive Manufacturing, 3D Print. Addit. Manuf., 2014, 1(1), p 2-3

3. W.E. Frazier, Metal Additive Manufacturing: A Review, $J$. Mater. Eng. Perform., 2014, 23, p 1917-1928

4. J.R. Davis, Aluminum and Aluminum Alloys, ASM Handbook, ASM International, Materials Park, 1999

5. E. Brandl, U. Heckenberger, V. Holzinger, and D. Buchbinder, Additive Manufactured AlSi10Mg Samples Using Selective Laser Melting (SLM): Microstructure, High Cycle Fatigue, and Fracture Behavior, Mater. Des., 2012, 34, p 159-169

6. G.P. Dinda, A.K. Dasgupta, and J. Mazumder, Evolution of Microstructure in Laser Deposited Al-11.28\%Si Alloy, Surf. Coat. Technol., 2012, 206, p 2152-2160

7. N.T. Aboulkhair, N.M. Everitt, I. Ashcroft, and C. Tuck, Reducing Porosity in AlSi10Mg Parts Processed by Selective Laser Melting, Addit. Manuf., 2014, 1-4, p 77-86

8. M. Nowak and N. Hari-Babu, Novel Grain Refiner for Hypo and Hyper-Eutectic Al-Si Alloys, LMT 2011 Conf paper, Materials Science Form, Vol 690, 2011, p 49-52.

9. M.C. Flemings, Solidification Processing, Metall. Mater. Trans. $B, 1974, \mathbf{5}, \mathrm{p} 2121-2134$

10. I. Rosenthal, A. Stern, and N. Frage, Microstructure and Mechanical Properties of AlSi10Mg Parts Produced by the Laser Beam Additive Manufacturing (AM) Technology, Metallogr. Microstruct. Anal., 2014, 3, p 448-453

11. K. Kempen, L. Thijs, J. Van Humbeeck, and J.P. Kruth, Mechanical Properties of AlSi10Mg Produced by Selective Laser Melting, Phys. Proced., 2012, 39, p 439-446

12. G.M. Miguel, AlSi10Mg Parts Produced by Selective Laser Melting (SLM), 2013, KU Leuven. Erasmus Stage/Universidad Carlos III de Madrid

13. M. Gupta and S. Ling, Microstructure and Mechanical Properties of Hypo/Hyper-Eutectic Al-Si Alloys Synthesized Using a NearNet Shape Forming Technique, J. Alloys Compd., 1999, 287, p 284-294

14. M. Gupta, C. Lane, and E.J. Lavenia, Microstructure and Properties of Spray Atomized and Deposited Al-7SiSiCp Metal Matrix Composites, Scr. Metall. Mater., 1992, 26, p 825

15. J. Hatch, Aluminum: Properties and Physical Metallurgy, American Society for Metals, Cleveland, 1984

16. A.E.W. Jarfors, Solidification Behaviour of $\mathrm{Al}-7 \% \mathrm{Si}-0.3 \% \mathrm{Mg}$ During Rotary Spray Forming, J. Mater. Sci., 1998, 33(15), p 3907-3918

17. D.W. Heard, S. Brophy, and M. Brochu, Solid Freeform Fabrication of $\mathrm{Al}-\mathrm{Si}$ Components via the CSC-MIG Process, $C M Q$, 2012, 51(3), p 301-312

18. D. Drouin, A.R. Couture, D. Joly, X. Tastet, V. Aimez, and R. Gauvin, CASINO V2. 42-A Fast and Easy-to-Use Modeling Tool for Scanning Electron Microscopy and Microanalysis Users, Scanning, 2007, 29, p 92-101 
19. M. Gäumann, S. Henry, F. Cléton, J.D. Wagnière, and W. Kurz, Epitaxial Laser Metal Forming: Analysis of Microstructure Formation, Mat. Sci. Eng. A, 1999, 271(1-2), p 232-241

20. H.B. Dong and P.D. Lee, Simulation of the Columnar-toEquiaxed Transition in Directionally Solidified Al-Cu Alloys, Acta Mater., 2005, 53(3), p 659-668

21. G.P. Dinda, A.K. Dasgupta, and J. Mazumder, Laser Aided Direct Metal Deposition of Inconel 625 Superalloy: Microstructural Evolution and Thermal Stability, Mater. Sci. Eng., A, 2009, 509(1-2), p 98-104

22. E. Scheil, Bemerkungen Zur Schichtkristallbildung (Comments on the Layer Crystal Formation), Z. Metallkd., 1942, 34, p 70-72 (in German)
23. H.D. Brody and M.C. Flemings, Solute Redistribution in Dendritic Solidification, Trans. AIME, 1966, 236, p 615-623

24. T.W. Clyne and W. Kurz, Solute Redistribution During Solidification with Rapid Solid State Diffusion, Metall. Trans. A, 1981, 12(6), p 965-971

25. E. Ogris, Development of Al-Si-Mg Alloys for Semi-solid Processing and Silicon Spheroidization Treatment (SST) for Al-Si Cast Alloys, Shaker Verlag GmbH, Germany, 2002

26. S.P. Nikanorov, M.P. Volkov, V.N. Gurin, Y.A. Burenkov, L.I. Derkachenko, B.K. Kardashev, L.L. Regel, and W.R. Wilcox, Structural and Mechanical Properties of Al-Si Alloys Obtained by Fast Cooling of a Levitated Melt, Mater. Sci. Eng., A, 2005, 390, p 63-69 